\title{
Do feature importance and feature relevance differentially influence lexical semantic knowledge in individuals with aphasia?
}

\author{
Lucia Scheffel \\ Department of Special Education and Communication Disorders, University of Nebraska at Omaha, NE, United States
}

\begin{abstract}
Purpose: This study investigated two classifications of semantic features, feature importance and feature relevance, to verify if they differentially influenced lexical semantic knowledge in individuals with aphasia.
\end{abstract}

Methods: A sorting task was utilized with 20 volunteer participants with aphasia to investigate the semantic processing involved in the association of semantic features with their appropriate nouns. A corpus of 18 nouns was displayed in front of each participant in groups of three along with a card containing the word "UNRELATED." The participants were verbally instructed to sort decks of cards into one of the four designated piles. The semantic features on the cards were rated as high, mid, and low importance and high, mid, and low relevance.

Results: The participants sorted high importance features with their nouns more accurately than they did mid and low importance features. Feature relevance did not differentially influence noun-feature association. These results indicated that the ability of individuals with aphasia to associate features with their nouns is influenced by levels of feature importance.

Conclusions: Lexical semantic knowledge in individuals with aphasia is influenced by high versus low level of feature importance and the effect does not extend to a mid level of importance. The classification of feature relevance did not differentially influence the ability of individuals with aphasia to associate features with their appropriate nouns. In addition, participants who were less affected by aphasia performed better in the naming and feature comprehension tasks.

Keywords: Aphasia, Lexical-semantic knowledge, Semantic features

\section{INTRODUCTION}

Difficulty comprehending and retrieving words associated with aphasia may arise from the dysfunction of various aspects of the cognitive structure and processing function of the semantic memory in the brain [1]. Semantic memory is a system that supports long-term knowledge of word meaning, also known as lexical semantic knowledge. It has been widely acknowledged that lexical semantic knowledge is composed of semantic features [2-5]. The employment of semantic features in aphasia therapy seems to be one variable that helps to improve naming performance of individuals with aphasia [6-8], although the results of treatments involving semantic features showed improvement of word retrieval when using trained and untrained items, it did not facili-

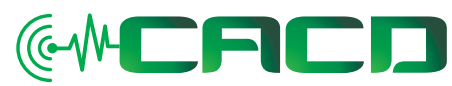

Received: April 24, 2021

Revision: September 24, 2021

Accepted: November 30, 2021

Correspondence:

Lucia Scheffel

Department of Special Educations and Communication Disorders, University of Nebraska at Omaha, Roskens Hall, 6001 Dodge Street, Omaha, NE 68182, United States

Tel: +402-554-3405

Fax: +402-554-3572

E-mail: Ischeffel@unomaha.edu

(C) 2021 The Korean Association of SpeechLanguage Pathologists

This is an Open Access article distributed under the terms of the Creative Commons Attribution NonCommercial License (https://creativecommons.org/ licenses/by-nc/4.0/) which permits unrestricted noncommercial use, distribution, and reproduction in any medium, provided the original work is properly cited. 
tate generalization during conversation. An important, yet unanswered question about semantic features is whether they are cognitively represented and organized in a particular way that would enhance lexical semantic knowledge in individuals with aphasia in order to facilitate word retrieval during contextual conversation.

Few researchers have investigated the role of feature classification in individuals with aphasia $[9,10]$. These authors support use of feature importance as the method of scaling lexical semantic knowledge. Feature importance is defined as how important a feature is in defining a concept [2]. A promising alternative for feature importance is feature relevance. According to Sartori and colleagues [11], feature relevance represents the combination of features that most identify the concept (i.e., dominant features) and, at the same time, discriminate it from other similar concepts (i.e., distinctive features). Based on this combination of attributes (dominance/ distinctiveness) our assumption is that feature relevance could possibly represent a better way to increase lexical semantic knowledge in individuals with aphasia.

The results of a pilot study by Scheffel [12] showed that organizing features by relevance did create a different hierarchy than the organization by importance in adults with no history of disorders in cognition and language. Therefore, investigating which feature classification, importance or relevance, best influenced the lexical semantic knowledge of individuals with aphasia could help us determine what type of semantic features could be used in an attempt to facilitate lexical retrieval in the aphasia rehabilitation process.

\section{Feature Importance versus Feature Relevance Studies Feature Importance}

Hampton [2,13] investigated the importance of semantic features for defining lexical-concepts through a series of surveys completed by adults without brain damage. He suggested that features can be systematically ranked for importance in defining a lexical-concept. For example, high importance features are those more commonly identified with a concept (e.g., "four legs" would be rated as a high importance feature for "cat"). Hampton's work [2] showed that when participants ranked features for their importance in defining a lexical-concept there was a significant relationship between the mean ranked importance of features and the frequency with which the features were produced.

Germani and Pierce [9] investigated feature importance in 13 adults with right hemisphere damage, 13 adults with left hemisphere damage and aphasia, and 13 adults with no history of disorders in cognition and language. A sorting task involving nouns with high, mid, and low frequency-of-occurrence and high and low importance features of each noun was employed. The results showed that the individuals with brain damage identified high importance features across all levels (e.g., high, mid, and low) of frequency of occurrence nouns as accurately as did the healthy individuals. Conversely, the individuals with brain damage were impaired in the identification of low importance features for mid and low frequency of occurrence nouns. Moreover, the results demonstrated that the ability to identify low-importance features was significantly correlated with naming and auditory comprehension skills. Mason-Baughman and Wallace [10] investigated the relationship between feature knowledge and the ability to choose among semantically related and unrelated foils in individuals with aphasia. Tasks for choosing among semantically unrelated and related foils and sorting tasks involving common and distinctive features controlled for mid and low semantic features importance were completed by 14 individuals with aphasia. The authors discovered that the individuals with aphasia who had difficulty choosing among semantically related foils had significantly more difficulty identifying low importance and distinctive features than those who were able to choose among semantically related foils. The authors also reported significant correlations between scores on tests of comprehension and distinctive feature identification, suggesting that distinctive feature knowledge contributes to the integrity of semantic representation in people with aphasia.

\section{Feature Relevance}

Sartori and Lombardi [14] proposed a model of semantic knowledge that measured the relevance of semantic features for a lexical-concept. They defined feature relevance as the extent to which a feature defines the lexical-concept and differentiates it from similar lexical-concepts. For instance, "has a trunk" was considered a semantic feature of high relevance for the lexical-concept "elephant" because most of the participants used it to define "elephant", whereas the same feature was seldom used to define other concepts. To the contrary, "has four legs" was a low relevance feature because few participants used it to define elephant although it was used to define other concepts. The authors used the semantic relevance model to investigate naming responses in seven individuals with category specific impairments (i.e., living versus nonliv- 
ing). Their results suggested that when living and nonliving categories were equated on levels of relevance, the categoryspecific deficits disappeared. Namely, no differences were detected between the naming of living and nonliving things when relevance was matched across categories. As a result, the authors stated that category effects emerge from differences in the relevance of features, and that lexical-concepts are better retrieved when semantic features with higher relevance are activated.

According to Lombardi and Sartori [15], feature relevance is the result of two components. The first component, dominance, is a measure of how frequently a feature is used in defining a concept. The second component, distinctiveness, is the extent to which a feature is characteristic of only a few examples of a category. Feature relevance scores are high when a semantic feature is often used in defining a concept (high dominance), but seldom mentioned in defining other concepts within the same category (high distinctiveness).

Sartori and colleagues [11] investigated the effect of feature relevance on a naming-to-description task with 15 patients with dementia of the Alzheimer type (DAT) and semantic disorders. They were asked to retrieve the name of a concept after hearing a sentence that described the target concept. The sentences included a set of three semantic features. For example, the sentence "has a handle, has two wheels and has two pedals" was presented orally to the participants, who were required to retrieve the name "bicycle." The authors reported a significant correlation between semantic relevance and accuracy, and concluded that semantic relevance was a good predictor of name retrieval accuracy in a name-to-description task.

\section{Lexical-Semantic Knowledge in Individuals with Aphasia}

One of the main components of treatment for individuals with aphasia is to improve their lexical-semantic knowledge. Several single-subject studies have reported the use of semantically based therapy as a treatment for word retrieval impairments in aphasia $[6,8,16,17]$. Outcomes of these studies have shown improved confrontation naming of trained and untrained items, but results have varied regarding maintenance of treatment effects and generalization to connected speech. In addition, the treatment approaches cited above were developed with minimal consideration regarding a systematic organization of semantic features which could ultimately help enhance lexical semantic knowledge facilitating naming skills. Therefore, there is still a need to investigate whether individu- als with aphasia are influenced by the organization of different types of semantic features that could be eventually used in the treatment of words retrieval deficits.

The existing literature suggests that some individuals with aphasia demonstrate category-specific deficits that could be explained by differences in feature relevance [14]. Some studies have considered the use of semantic features as an important factor in the improvement of lexical-semantic knowledge in individuals with aphasia $[6,8,16,17,20]$. In fact, the employment of a semantic features approach seems to be an effective treatment procedure because it improves lexical retrieval by accessing lexical-semantic networks [21]. Namely, it is assumed that by activating the lexical-semantic networks surrounding the target word, that target itself will be sufficiently activated to allow retrieval of the word [6,8]. Although Boyle and Coelho [6] and Coelho et al. [8] found that participants with aphasia benefited from the semantic feature analysis treatment by improving lexical retrieval, the authors used a broad group of features in their studies. Accordingly, the organization of these features may have influenced their effect on lexical-semantic knowledge in persons with aphasia.

Organization of features by importance has some influence on individuals with aphasia $[9,10]$. However, the organization of features based on relevance had not been studied yet in individuals with aphasia prior to the current project. Based on the work of Sartori and Lombardi [14], Sartori, Lombardi and Mattiuzzi [11] and Lombardi and Sartori [15], relevance may represent a promising alternative to feature importance [9]. The results of our pilot study [12] indicated that organizing features by relevance does create a different lexical hierarchy than organization by importance. Therefore, we aimed to determine whether or not lexical semantic knowledge in individuals with aphasia would be influenced by different levels of semantic feature relevance. Specifically, we examined whether individuals with aphasia were more accurate to feature importance or feature relevance ratings.

\section{METHODS}

\section{Participants}

Twenty adults, ten males and ten females, with aphasia resulting from one $(\mathrm{N}=18)$ or two $(\mathrm{N}=2)$ unilateral cerebrovascular accidents (CVA) participated in this study. Paticipants' ages ranged between 43 and 83 years of age ( $M=63.6$ years, $S D=11.7)$. Education level ranged from high school diploma to doctoral degree. All the participants were right-handed na- 
tive speakers and readers of American English and had no medical history of mental deterioration such as Parkinson's disease or dementia based on information from the referring speech-language pathologist and confirmation from each participant or family member. Participants were between 5 and 199 months post onset of aphasia $(M=70.5, S D=60.9)$ and were classified as having fluent or non-fluent aphasia based on each participant's responses during the Conversational and Expository Speech components of the Boston Diagnostic Aphasia Examination Third Edition (BDAE-3) [27]. These measures included: (1) Basic Word Description, (2) Commands, and (3) Complex Ideational Material. Also, the short version of the Boston Naming Test (BNT-2) [28] was used to measure the participants' naming abilities. Aphasia

Table 1. Participant descriptive data

\begin{tabular}{|c|c|c|c|c|c|}
\hline Participant & Sex $x^{a}$ & $\begin{array}{l}\text { Aphasia } \\
\text { Type }^{\mathrm{b}}\end{array}$ & $\mathrm{Age}^{\mathrm{c}}$ & $\mathrm{TPO}^{\mathrm{d}}$ & Education \\
\hline 1 & M & NF & 72 & 199 & $\mathrm{BD}$ \\
\hline 2 & $\mathrm{~F}$ & NF & 45 & 65 & $\mathrm{DD}$ \\
\hline 3 & M & $\mathrm{F}$ & 60 & 21 & $\mathrm{DD}$ \\
\hline 4 & $\mathrm{~F}$ & $\mathrm{~F}$ & 65 & 5 & $\mathrm{BD}$ \\
\hline 5 & $\mathrm{~F}$ & NF & 58 & 6 & HSD \\
\hline 6 & $\mathrm{~F}$ & $\mathrm{~F}$ & 60 & 14 & $\mathrm{BD}$ \\
\hline 7 & M & NF & 57 & 64 & $A D$ \\
\hline 8 & M & $\mathrm{F}$ & 78 & 109 & $\mathrm{DD}$ \\
\hline 9 & $\mathrm{~F}$ & $\mathrm{~F}$ & 43 & 76 & $A D$ \\
\hline 10 & M & NF & 49 & 95 & $\mathrm{BD}$ \\
\hline 11 & M & $\mathrm{F}$ & 83 & 198 & $\mathrm{MD}$ \\
\hline 12 & M & NF & 78 & 17 & HSD \\
\hline 13 & $\mathrm{~F}$ & $\mathrm{~F}$ & 67 & 121 & HSD \\
\hline 14 & $\mathrm{~F}$ & $\mathrm{~F}$ & 56 & 103 & HSD \\
\hline 15 & M & NF & 66 & 135 & $\mathrm{BD}$ \\
\hline 16 & $\mathrm{~F}$ & NF & 77 & 18 & HSD \\
\hline 17 & $\mathrm{~F}$ & NF & 66 & 50 & HSD \\
\hline 18 & M & $\mathrm{F}$ & 67 & 93 & $\mathrm{BD}$ \\
\hline 19 & $\mathrm{~F}$ & NF & 49 & 16 & $\mathrm{BD}$ \\
\hline 20 & M & $\mathrm{F}$ & 77 & 6 & HSD \\
\hline Mean & & & 63.6 & 70.5 & \\
\hline SD & & & 11.7 & 60.9 & \\
\hline Range & & & 40 & 194 & \\
\hline
\end{tabular}

${ }^{\mathrm{a} M}$, male, $\mathrm{F}$, female; ${ }^{\mathrm{b}} \mathrm{F}$, fluent aphasia, NF, nonfluent aphasia; ${ }^{\mathrm{c}} \mathrm{Age}$ in years; dTime post-onset in months.

$H S D$, high school diploma, $A D$, associate degree, $B D$, bachelor degree, MD, master degree, $\mathrm{DD}$, doctoral degree. severity was obtained from the Language Competency Index which comprises of above subtests as well as the Grammatical Rating Form. Inter-judge agreement by two speech-language pathologists was 100 percent. Table 1 contains relevant identifying information for each participant. Table 2 contains the scores on independent measures for each subject.

The participants with aphasia were recruited from local stroke groups, hospitals, out-patient clinics, and nursing homes, and agreed to participate in the study by signing a consent form. The presence of aphasia was diagnosed by the referring speech-language pathologist and confirmed by the investigator based on results of language tests cited above. Also, it was required that the onset of the most recent stroke should be at least one month prior to participation, and that

Table 2. Participants' Scores on Subtests of the Boston Diagnostic Aphasia Examination-3 Shorted Version and Boston Naming Test-2 Shorted Version

\begin{tabular}{|c|c|c|c|c|c|}
\hline Participants & BWD $^{\mathrm{a}}$ & $\mathrm{COM}^{\mathrm{b}}$ & $\mathrm{CIM}^{\mathrm{c}}$ & $\mathrm{BNT}^{\mathrm{d}}$ & $\mathrm{LCl}^{\mathrm{e}}$ \\
\hline 1 & 13 & 6 & 4 & 10 & 43 \\
\hline 2 & 16 & 9 & 6 & 11 & 74 \\
\hline 3 & 16 & 10 & 6 & 12 & 87 \\
\hline 4 & 16 & 10 & 6 & 9 & 90 \\
\hline 5 & 16 & 8 & 5 & 8 & 67 \\
\hline 6 & 14 & 6 & 6 & 7 & 77 \\
\hline 7 & 14 & 7 & 4 & 6 & 35 \\
\hline 8 & 16 & 10 & 6 & 10 & 93 \\
\hline 9 & 16 & 9 & 6 & 13 & 77 \\
\hline 10 & 13 & 2 & 4 & 5 & 27 \\
\hline 11 & 13 & 4 & 3 & 0 & 16 \\
\hline 12 & 10 & 4 & 3 & 3 & 30 \\
\hline 13 & 16 & 7 & 6 & 9 & 70 \\
\hline 14 & 14 & 5 & 4 & 7 & 43 \\
\hline 15 & 10 & 6 & 2 & 2 & 22 \\
\hline 16 & 16 & 10 & 6 & 6 & 75 \\
\hline 17 & 16 & 8 & 6 & 5 & 55 \\
\hline 18 & 16 & 8 & 6 & 15 & 90 \\
\hline 19 & 16 & 9 & 5 & 9 & 56 \\
\hline 20 & 16 & 10 & 6 & 13 & 95 \\
\hline Mean & 14.8 & 7.4 & 5.0 & 8.0 & 62.1 \\
\hline SD & 1.9 & 2.3 & 1.3 & 3.9 & 26.1 \\
\hline Range & 6 & 8 & 4 & 15 & 79 \\
\hline
\end{tabular}

${ }^{a}$ Basic Word Discrimination $(\max =16)$; ' ${ }^{\text {b }}$ Commands $(\max =10)$; ' Complex Ideational Material (max=6); 'Boston Naming Test $(\max =15)$; ' Language Competency Index (percentile max=100). 
the participants had adequate visual and hearing acuity for perfoming the experimental tasks as detemined by their ability to pass the screening training tasks.

In addition, in order to establish basic word comprehension and be accepted into this investigation, each participant was required to pass a screening test. Six sets of three nouns taken from the experimental task were used for the screening test. One at a time, nouns were auditorily presented by the examiner as the participant views three written nouns displayed in a vertical array. The screening test consisted of having each participant point to the target noun in the array in front of him or her. Responses were scored as either correct $(+)$ or incorrect (-). A score of $5 / 6$ or higher had to be achieved by a participant in order to be included in this investigation. No one was excluded from the study based on this criterion.

\section{Development of Stimuli}

Nouns chosen as stimuli consisted of 9 low $(<8 /$ million $)$ and 9 mid (10-25/million) frequency of occurrence nouns based on Francis and Kucera [29] and their semantic features obtained in the pilot study [12]. The semantic features were rated as high, mid and low importance (HI, MI, LI) and high, mid and low relevance (HR, MR, LR) [12]. For example, the semantic features "red skin" for the noun "apple" was rated as low importance (LI) and high relevance (HR). The Appendix shows the thirty nouns as well as their semantic features for each combination of importance and relevance condition (high, mid, and low). The following criteria were used in order to select the importance and relevance features in this study: (1) features with relevance and importance ratings at or near the boundaries were removed; (2) features were identified near the middle of relevance and importance ratings (e.g., 1.3, 1.4, or 2.5, 2.6, or 3.5, 3.6. 3.7); (3) when a noun had more than one feature in a condition (high, mid and low), one or more noun-feature pairs were removed; (4) if more than ten features remained after these steps, then features were randomly removed until ten remained. The HR-LI condition had only 10 features, consequently all of them were selected. Also, the HRMI condition had only 13 features, thus 10 of 13 nouns were chosen. Although we tried to follow the above criteria, there were two features in one condition for the nouns "apple" (HRLI), "soap" (HR-LI), and "stove" (HR-MI). Because the intention in the experimental task was to present three nouns at a time to the participants, we decided to randomly eliminate two nouns from the corpus of 20 nouns investigated in the pilot study [12]. This way the investigation had a corpus of 18 frequency nouns. In addition, four unrelated features were added to each of the six sets of three nouns. The unrelated features were taken from the pilot study [12]. As a result, the investigation had a total of 83 semantic features. To avoid displaying nouns from similar categories, the 18 nouns were arranged in two pseudo-randomized orders. For example, "apple", "blouse", and "butterfly" were selected for a set as opposed to "apple", "lemon" and "strawberry." All the nouns were typed on 5 " $\times 8$ " white index cards using Times Roman 36 -font in capital letters. The semantic features for each noun and also for the unrelated features were typed on 4 " $\times 6$ " white index cards using Times New Roman 36-font lower case letters.

\section{Procedures}

This investigation was divided into two stages: (1) training and experimental task, and (2) feature comprehension task.

\section{Stage 1:}

Participants were engaged in a training task prior to the experimental task. In the training task, participants were verbally presented with nine nouns taking from a corpus of stimuli used by Germani [25]. These nouns were not used in the experimental task. The nouns were shown in groups of three accompanied by the word labeled "UNRELATED." The participants were instructed to sort a deck of features into one of the three nouns or into the unrelated word if the feature does not match with any of the nouns presented. All features were said aloud by the investigator. Responses for the training task were also scored as correct (+) or (-) incorrect. Participants were required to achieve a minimum of 7/9 correct responses to move on to the experimental task. If this score was not achieved, then participants were reinstructed and presented with a demonstration of the sorting task before attempting it the second time.

The experimental task involved a sorting task. Each participant viewed 6 groups of three nouns and the word "unrelated." A corpus of 18 nouns was displayed in front of each participant in groups of three together with the word "UNRELATED." These nouns were presented in random order with the consideration that only one noun from a specific category was in each set of three nouns. The participants were given a deck of cards containing features corresponding to the nouns and to the unrelated category, and were verbally instructed to sort the deck of cards into one of the four designated piles. Each feature word or phrase was said aloud by the examiner. 
Scheffel L, et al. Semantic features and lexical semantic knowledge

The size of the deck per set of nouns varied, but there was an average of 18 cards in each deck. After a card or phrase was sorted into one of the four piles, it was removed from the table. This avoided having participants to determine a pattern of features in any given pile. This sorting task was repeated 6 times for each participant for a total of 18 nouns. Sorting responses in this stage were scored as either correct $(+)$ or incorrect (-).

\section{Stage 2:}

The second stage of the procedures involved a test of comprehension of mid and low levels of importance and relevance features. These features were taken from all conditions investigate MI-MR, MI-LR, LI-MR, and LI-LR as shown in Appendix. The comprehension task consisted of 12 presentations of three features from the mid and low level of importance and relevance. That is, each row contained three words or three phrases. Each participant was instructed to point to the feature that was verbally requested by the investigator. The sheets of paper were removed as soon as the participant provided the response and the same was scored correct $(+)$ or incorrect $(-)$.

\section{Statistical Analysis}

This study had three major variables. The dependent variable was the performance score for correct features sorted with target words. The performance score was a ratio scale from zero to 20. Two independent variables, feature importance and feature relevance, were measured each by high, mid and low rating levels. Since performance was a ratio scale and the study aimed to compare the differences between three levels of two groups of features (e.g., feature importance and feature relevance), analysis of variance (ANOVA) was thought to be a good fit.

\section{RESULTS}

The purpose of the present study was to investigate what kind of semantic features best influence lexical semantic knowledge in individuals with aphasia. Two types of feature classification were examined: relevance and importance. In addition, we aimed to find out whether individuals with aphasia are more sensitive to levels (e.g., high, mid, and low) of feature important or feature relevance.

Data were first analyzed to look at feature importance versus feature relevance means, standard deviations, and range
Table 3. Number of features, Means, Standard Deviation and Range Scores for High, Mid, and Low Feature Importance and Feature Relevance ( $N=20)$

\begin{tabular}{lcccccccc}
\hline & \multicolumn{3}{c}{ Importance } & & \multicolumn{3}{c}{ Relevance } \\
\cline { 2 - 3 } \cline { 7 - 9 } & Low & Mid & High & & Low & Mid & High \\
\hline $\mathrm{N}$ & 28 & 29 & 26 & & 27 & 29 & 27 \\
Mean & 14.7 & 16.1 & 17.3 & & 15.2 & 15.8 & 17.0 \\
SD & 3.9 & 3.2 & 3.4 & & 4.4 & 3.5 & 2.7 \\
Range & 14 & 12 & 17 & & 17 & 12 & 9 \\
\hline
\end{tabular}

scores for the performance of 20 participants with aphasia in the sorting experimental task. Table 3 contains the descriptive analyses for each separate condition of high, mid, and low levels of feature importance and feature relevance.

Results from the experimental task were computed using SPSS software 20.0. A two-way between-subjects ANOVA was used to examine whether the three different levels (e.g., low, mid and high) of importance and relevance means differ or not, and to analyze whether there was an interaction between feature importance and feature relevance levels.

Results for the Levene's Test indicated that equal variances among high, mid and low groups were assumed ( $p=0.09)$. Therefore, homogeneity of variances was assumed at $95 \%$ confidence level. In addition, analyses of multivariate normality showed Skewness and Kurtosis values near equal zero reflecting a normal distribution among the means. Therefore, multivariate normality and similar sample size in the groups were assumed.

Results of the two-way between subjects ANOVA showed a statistically significant main effect for feature importance $F(2$, $82)=3.36, p=0.04$. This result indicated that the ability of individuals with aphasia to associate features with their nouns is influenced by the importance of semantic features. The study results showed that the strength of relationship between feature importance and performance in the sorting task was weak, with $\eta^{2}=0.083<0.2$ (small effect size). However, a power of $62 \%$ ( $\beta=0.62)$ was observed, minimizing the risk that the null hypothesis was true.

Bonferroni post-hoc analysis was used to determine which pairs of means were and were not statistically significant among the different levels of feature importance. Bonferroni results showed that only "low" and "high" feature importance were statistically significant different $(p=0.03)$, indicating different means between these two groups. For the post-hoc analysis among the levels of "high" and "mid" $(p=0.69)$, and "low and "mid" ( $p=0.47)$ feature importance, Bonferroni test 
did not provide statistically significant results, indicating equal means among this levels of feature importance. No statistically significant main effect of feature relevance and performance in the sorting task was found $F(2,82)=1.70, p=0.18$.

In addition, the comparison between feature importance and feature relevance failed to reveal an interaction effect $(F=0.14 ; d f=4 ; p=0.96)$. This result means that the type of feature, importance versus relevance, had no effect on the participants' performance in the sorting task. Both classifications seemed to be equally preserved in the brain of participants with aphasia. See Table 4 for the ANOVA results.

To investigate the associative relationship between feature knowledge and comprehension and naming skills, Pearson correlations were computed among scores from the three levels in the experimental conditions of feature importance (HI, MI, and LI); the three levels of feature Relevance (HR, MI, LR); the Language Competency Index (LCI) of the BDAE-3 Short Form; the BNT-2 and the Feature Comprehension Task (FC) derived from the experimental stimuli. Table 5 shows the correlation matrix.

Significant correlations were observed among the Boston

Table 4. Results of the Two-Way Between-Subjects ANOVA for Feature Importance and Feature Relevance $(\mathrm{N}=20)$

\begin{tabular}{lcccccc}
\hline Source & df & $\begin{array}{c}\text { Partial } \\
\text { Eta } \\
\text { Square }\end{array}$ & $\begin{array}{c}\text { Mean } \\
\text { Square }\end{array}$ & F & Significance & $\begin{array}{c}\text { Observed } \\
\text { Power }\end{array}$ \\
\hline Importance & 2 & 0.083 & 44.1 & 3.36 & 0.04 & 0.62 \\
Relevance & 2 & 0.046 & 23.4 & 1.78 & 0.17 & 0.36 \\
$\begin{array}{l}\text { Importancex } \\
\text { Relevance }\end{array}$ & 4 & 0.008 & 1.86 & 0.14 & 0.96 & 0.78 \\
\hline
\end{tabular}

Naming Test (BNT), the LCI, and the Feature Comprehension (FC) task. Moreover, all the experimental conditions correlated significantly with the BNT and the LCI. Of interest was the fact that FC did not correlate significantly with high importance $(r=0.38)$, low importance $(r=0.34)$ and high relevance features $(r=0.25)$, but did correlate significantly with mid importance $(r=0.65)$, mid relevance $(r=0.47)$, and low relevance $(r=0.47)$. All these significant correlations indicated that the participants who are less affected by aphasia tend to perform better in the language and experimental conditions tasks. Those who had difficulty sorting semantic features with their appropriate nouns also demonstrated difficulty performing comprehension and naming tasks.

\section{DISCUSSION}

\section{Levels of Feature Importance and Lexical Semantic Knowledge}

The results revealed that different levels of feature importance significantly influence feature-noun association task in those individuals with aphasia. The same was not observed for feature relevance. High importance features were identified with their appropriate nouns more accurately than were low importance features. However, a statistically significant result was not observed in the comparison of high and mid, and mid and low importance features, meaning that two set of levels of feature importance were identified by individuals with aphasia with similar degrees of accuracy.

Past studies further support a distinction between "high versus low" importance features. In research aiming to investigate the ability of individuals with aphasia to associate fea-

Table 5. Correlations among Participant's Scores ( $N=20)$

\begin{tabular}{|c|c|c|c|c|c|c|c|c|c|}
\hline & $\mathrm{HI}$ & $\mathrm{MI}$ & LI & HR & MR & LR & BNT & $\mathrm{LCl}$ & FC \\
\hline $\mathrm{HI}$ & $\ldots$ & $0.62^{* *}$ & $0.71^{* *}$ & $0.57^{* *}$ & $0.76^{* *}$ & $0.82^{* *}$ & $0.51^{*}$ & $0.49^{*}$ & 0.38 \\
\hline $\mathrm{Ml}$ & & $\ldots$ & $0.72^{* *}$ & $0.57^{*}$ & $0.90^{* *}$ & $0.86^{* *}$ & $0.77^{* *}$ & $0.69^{* *}$ & $0.65^{* *}$ \\
\hline $\mathrm{LI}$ & & & $\ldots$ & $0.87^{* *}$ & $0.82^{* *}$ & $0.75^{* *}$ & $0.53^{*}$ & $0.46^{*}$ & 0.34 \\
\hline$H R$ & & & & $\ldots$ & $0.52^{*}$ & $0.57^{* *}$ & $0.52^{*}$ & $0.53^{*}$ & 0.25 \\
\hline $\mathrm{MR}$ & & & & & $\ldots$ & $0.80^{* *}$ & $0.67^{* *}$ & $0.51^{*}$ & $0.47^{*}$ \\
\hline LR & & & & & & $\ldots$ & $0.60^{* *}$ & $0.63^{* *}$ & $0.58^{* *}$ \\
\hline BNT & & & & & & & .. & $0.81^{* *}$ & $0.63^{* *}$ \\
\hline $\mathrm{LCl}$ & & & & & & & & $\ldots$ & $0.59^{* *}$ \\
\hline FC & & & & & & & & & $\ldots$ \\
\hline
\end{tabular}

HI, high importance; MI, mid importance; LI, low importance; HR, high relevance; MR, mid relevance; LR, low relevance; BNT, boston naming test; LCl, language competency index; FC, feature comprehension.

${ }^{*} p<.05$ level (two-tailed test); ${ }^{* *} p<.01$ level (two-tailed test). 
tures importance with high, mid, and low frequency of occurrence nouns, Germani and Pierce [9] found that individuals with aphasia identified high-importance semantic features in all three (high, mid, and low) frequencies of occurrence nouns with high levels of accuracy. However, those same individuals showed impaired identification of low-importance features for mid and low frequency of occurrence nouns. Mason-Baughman and Wallace [10] found that individuals with aphasia, who demonstrated difficulty choosing among semantically related foils, had significantly more difficulty matching low importance distinctive features with nouns when compared to matching high importance distinctive features with nouns.

The results of this study reinforce the notion that, when using tasks of associating features (e.g., sorting/matching) with a target noun, semantic feature knowledge in persons with aphasia is influenced by two main hierarchical levels of semantic features difficulty: high versus low importance $[9,10]$. In addition, the findings of the present study did not reveal a statistically significant difference between high and mid, and/ or mid and low importance feature levels. Mid importance level seems not to be strongly represented in the mind of individuals with aphasia. Namely, the hierarchy of semantic features knowledge was not extended to a third level of difficulty: mid level of importance. It should be noted that, although the finding in this study did not show a numerical trend between the levels of mid and low importance - that is, both conditions were equivalent in difficulty - visual inspection of the results clearly demonstrates that individuals with aphasia tend to perform better when associating/sorting mid important features with their appropriate nouns than low importance features. Although there was not as much variability in performance when compared to low versus high levels of feature importance, Table 3 suggests that the means for the levels of low and mid feature importance are indeed different. A statistically significant difference on hierarchy levels of relevance was not observed in the individuals with aphasia. Also, visual inspection analysis showed that the variability in performance among the levels of high, mid and low feature relevance is smaller when compared to the high, mid and low levels of importance.

\section{Feature Importance versus Feature Relevance and their Influence in the Lexical Semantic Knowledge of Individuals with Aphasia}

Difficulty understanding and producing language by individ- uals with aphasia may be related to deficits in the lexical semantic knowledge (e.g., semantic features) of concepts. Very few studies have investigated the role of semantic feature classification in the lexical semantic knowledge in people with aphasia $[9,10]$. Two types of feature classification were investigated in the present study: Relevance and importance.

According to Sartori and colleagues [11], feature relevance is defined as those features that most identify the concept and, at the same time discriminate it from other similar concepts. Lombardi and Sartori [15] proposed that feature relevance is the result of two components. The first component, dominance, is a measure of how frequently a feature is used in defining a concept. The second component, distinctiveness, is the extent to which a feature is characteristic of only a few examples of a category. Feature relevance scores are high when a semantic feature is often used in defining a concept (high dominance), but seldom mentioned in defining other concepts within the same category (high distinctiveness). On the contrary, Hampton [2] defined feature importance as how important a feature is in defining a concept. The author proposed that features could be systematically ranked for importance when lexically defining a semantic representation. He used a series of surveys completed by healthy adults to determine the importance of features. High importance features were those rated as very important to the definition of a concept and/or necessarily true of all possible exemplars of the representation. Low importance features were those rated as not true or usually true of the object.

The primary hypothesis of this study was that relevance and importance are different ways of classifying semantic features and, therefore, it was possible that individuals with aphasia could be more cognitively sensitive to one or the other. The findings support feature importance as the classification of features that best facilitates feature comprehension in individual with aphasia. The results from this study indicated a differential impairment of high importance versus low importance semantic features in participants with aphasia, supporting previous finings of Germani and Pierce [9]. A significant main effect of importance was also found that replicated the findings of Germani and Pierce [9] and Mason-Baughman and Wallace [10] in that high importance features were sorted more accurately then low-importance features.

Our results did not support relevance of features as a significant factor that contributes to the comprehensives of semantic representation in persons with aphasia. The fact that feature relevance did not significantly influence the ability of in- 
dividuals with aphasia to sort features with their appropriate nouns possible suggests that these individuals did not perceive the essential nature of feature relevance (e.g., the combination of common and distinctive features) to a concept. Mason-Baughman and Wallace [10] pointed out that the intact knowledge of features considered common to a semantic category is necessary in order to distinguish a target word from different semantic categories. On the other hand, knowledge of distinctive features may be required to distinguish the target from words within the same category. Namely, distinctive feature knowledge is necessary to process the subtle difference when choosing a target from an array of semantic related foils, but not always needed to choose a target from an array of semantic unrelated foils. Mason-Baughman and Wallace [10] found that individuals with aphasia who had difficulty choosing among semantic related foils were more impaired with the identification of low importance distinctive features when compared to high importance distinctive features and the identification of common features.

Features that have a strong distinctive component in nature might also play a role in how individuals with aphasia access lexical semantic knowledge from different categories. When we examined the two components of feature relevance separately (dominance and distinctiveness), it was noted that the features which had a low score of performance in the sorting task were those rated as having a low dominance component (e.g., low commonality) but at the same time a high distinctive component. Moreover, those same semantic features were also rated as having low or mid levels of importance. These results might inform us as to why relevance did not emerge as significant factor in the ability of individuals with aphasia to sort features with the target words. Perhaps, as stated by Mason-Baughman and Wallace [10], intact feature distinctiveness contributes to the comprehensiveness of lexical semantic knowledge in persons with aphasia. It is possible that the high distinctiveness component of feature relevance affected the ability of individuals with aphasia to sort features with target nouns. Therefore, an intact knowledge of distinctive features may be needed in order to have more robust feature knowledge and to be able to distinguish among items from same categories [10] and items from other broad categories.

It is also interesting to point out that the majority of semantic features which received a lower performance score in the sorting task were features belonging to the domain specific of "living things" and rated as highly distinctive. Randall et al. [26] claimed that there are few correlations among distinctive features and living things and that is why feature distinctive for certain concepts are more vulnerable to damage. After calculating the interaction between features and the domains of living and nonliving things, the authors found that the correlation strength among distinctive features was significantly greater for nonliving things than for living things. In addition, the results of a speed feature verification task showed that participants had slower reaction times for verifying the distinctive features of living thing when compared to the reaction times of distinctive features of nonliving things. The authors concluded that the weak correlations among living things with other features can affect the speed in which the meaning of a concept is activated, and therefore delay access and make performance more error-prone. The Randal et al. [26] findings help to explain why individuals with aphasia in the present study had more difficulty sorting relevant features than importance features with target nouns. Perhaps because semantic features tend to be distributed differently across concepts, it is more likely that feature importance captures the salience of any giving concept. That is, features that are important in nature will always have privileged access in the structure of the semantic system because they robustly facilitate access to activate meaning and recognition of a concept. On the contrary, the same is not always true for feature relevance because the "distinctive" component of it may be sometimes weakly correlated with other features, and therefore affect the strength of the relationship with the concepts.

The findings from this study support Germani and Pierce [9] and Mason-Baughman and Wallace [10] in determining that feature importance contributes to the comprehensiveness of semantic representation in individuals with aphasia. Also, the results of this study corroborate the Mason-Baughman and Wallace [10] study in that persons with aphasia who had impaired knowledge of highly distinctive features also showed decrease accuracy sorting features with target nouns.

\section{Feature Correlations and Language Skills}

The relationship of comprehension and naming abilities to feature knowledge was investigated using correlations. Scores on measures of comprehension from the BDAE-3 Language Competency Index correlated significantly with scores on experimental feature conditions knowledge. These findings suggest that individuals with aphasia who have intact feature representations, for both feature importance and feature relevance, have better overall language abilities. Consequently, improved feature knowledge helps them to successfully sort 
features to target nouns.

These results support previous findings from Germani and Pierce [9] and Mason-Baughman and Wallace [10] who found significant correlations among scores for auditory comprehension skills with low importance features [9], and with common and distinctive features of high and low importance [10]. Moreover, there were some correlations among the various scores for features conditions and features comprehension (FC) task. However, a meaningful pattern among these correlations was difficult to determine. It is likely that the nature of the feature comprehension task was too easy and did not capture the essence of the impairment.

The relationship between naming abilities and semantic feature knowledge was also significantly correlated. That is, individuals with aphasia who demonstrated a more intact lexical semantic knowledge were the ones who obtained a higher score on the Boston Naming Test. The findings of the present study also corroborate studies by Germani and Pierce [9] and Mason-Baughman and Wallace [10] who found statistically significant correlations among importance feature conditions and naming abilities.

\section{LIMITATIONS}

Although the findings of the present study support previous research by Germani and Pierce [9] and Mason-Baughman and Wallace [10], a larger sample size could increase the power of data analysis leading to results that support the current findings. In addition, a larger and more uniformed corpus of importance and relevance features (e.g., HI, MI, LI, HR, MR, and LR) could confirm or show different measures of effect size accounting for the real variance found among the different levels of feature importance and feature relevance. Data were also collected from a mixed representation of participants in terms of aphasia classification (e.g., fluent versus non-fluent) and severity. Five out of 20 participants obtained $87 \%$ or higher score (up to $100 \%$ ) in the Language Competency Index (LCI) of the Boston Diagnostic Aphasia Examination-3 Shorted Version. As a result, their level of performance of the experimental sorting task may have been closer to that of non-brain damaged individuals. An investigation testing more severely impaired fluent individuals with aphasia could show changes in the variability scores among the levels of feature importance and feature relevance leading to different mean results. Moreover, a sorting task has been used in all the previous studies that investigated the role of classification and organization of semantic features. Perhaps using a different task to investigate whether feature importance and feature relevance differently influence lexical semantic knowledge in individuals with aphasia would certify that the results are not task dependent. Finally, this is one of many studies investigating semantic knowledge in individuals with aphasia and needs to be considered in this broader context.

\section{CONCLUSION}

The findings of the present study suggest that individuals with aphasia are more sensitive to high versus low importance feature in sorting features with their correspondent nouns. That is, high importance features were identified more accurately than low importance features; however, mid importance features were not. Feature relevance was not shown to influence lexical semantic knowledge in a hierarchical manner. These results support previous research investigating how semantic features are cognitively classified and organized in the brain of individuals with aphasia. Semantic features can be organized according to their importance of features.

\section{REFERENCES}

1. Raymer AM, Rothi LJG. Impairments of word comprehension and production. In: R. Chapey (Ed.), eitor. Language intervention strategies in aphasia and related neurogenic communication disorders (pp. 607-631). Baltimore: Lippincott Williams \& Wilkins; 2008.

2. Hampton JA. Polymorphous concepts in semantic memory. Journal of Verbal Learning and Verbal Behavior. 1979;18: 441-461.

3. Caramazza A. How many levels of processing are there in lexical access? Cognitive Neuropsychology. 1997;14:177-208.

4. Dell GS, Schwartz MF, Martin N, Saffran EM, Gagnon DA. Lexical access in aphasic and nonaphasic speakers. Psychological Review. 1997;104:801-838.

5. Martin A. Functional neuroimaging of semantic memory. In: R. Cabeza \& A. Kingstone (Eds), eitors. Handbook of functional neuroimaging of cognition (pp. 149-190). Cambridge, MA:MIT Press; 2001.

6. Boyle M, Coelho CA. Application of semantic feature analysis as a treatment for aphasic dysnomia. American Journal of SpeechLanguage Pathology. 1995;4:94-98.

7. Boyle M. Semantic feature analyses treatment for anomia in two fluent aphasia symdromes. American Journal of Speech-Language Pathology. 2004;13:236-249.

8. Coelho CA. McHugh RE, Boyle M. Semantic feature analysis as a treatment for aphasic dysnomia: A replication. Aphasiology. 2000;14:133-142.

9. Germani MJ, Pierce R. Semantic attribute knowledge in adults 
with right and left hemisphere damage. Aphasiology. 1995;9:1-22.

10. Mason-Baughman MB, Wallace SE. Semantic feature knowledge in persons with aphasia: The role of commonality, distinctiveness and importance. Aphasiology. 2013;27:364-380.

11. Sartori G, Lombardi L, Mattiuzzi L. Semantic relevance best predicts normal and abnormal name retrieval. Neuropsychologia. 2005;43:754-770.

12. Scheffel L. Do Feature Importance and Feature Relevance Represent Different Ways of Organizing Lexical-Semantic Knowledge? Unpublished manuscript, Kent State University - Kent. 2010.

13. Hampton JA. Inheritance of attributes in natural concept conjunctions. Memory and Cognition. 1987;15:55-71.

14. Sartori G, Lombardi L. Semantic relevance and semantic disorders. Journal of Cognitive Neuroscience. 2004;16:439-452.

15. Lombardi L, Sartori G. Models of relevant cue integration in name retrieval. Journal of Memory and Language. 2007;57:101-125.

16. Lowell S, Beeson PM, Holland AL. The efficacy of a semantic cuing procedure on naming performance of adults with aphasia. American Journal of Speech-Language Pathology. 1995;4:109-114.

17. Drew RL, Thompson CK. Model-based semantic treatment for naming deficits in aphasia. Journal of Speech, Language \& Hearing Research. 1999;42:972-989.

18. Kiran S, Thompson CK. Effect of typicality on online category verification of animate category exemplars in aphasia. Brain and Lan- guage. 2003;85:441-450.

19. Kiran S, Johnson L. Semantic complexity in treatment of naming deficits in aphasia: Evidence from well-defined categories. American Journal of Speech-Language Pathology. 2008;17:389-400.

20. Kiran S. Typicality treatment for naming deficits in aphasia: Why does it work? Perspectives on Neurophysiology and Neurogenic Speech and Language Disorders. 2008;18:6-14.

21. Massaro ME, Tompkins CA. Feature analysis for treatment of communication disorders in traumatically brain injured patients: An efficacy study. Clinical Aphasiology. 1992;22:245-256.

22. Goodglass H, Kaplan E, Barresi B. The Boston Diagnostic Aphasia Examination (3rd edition). Philadelphia. PA: Lippincot Williams \&Willkins; 2001.

23. Kaplan E, Goodglass H, Weintraub S. Boston Naming Test. Philadelphia: Lea \& Febiger; 1983.

24. Francis WN, Kucera H. Frequency analysis of English usage: Lexicon and grammar. Boston: Houghton Mifflin Company; 1982.

25. Germani MJ. Semantic attribute knowledge in adults with right and left hemisphere damage. Unpublished doctoral dissertation, Kent State University - Kent. 1992.

26. Randall B, Moss HE, Rodd JM, Greer M, Tyler LK. Distinctiveness and correlation in conceptual structure: Behavior and computational studies. Journal of Experimental Psychology: Learning, Memory and Cognition. 2004;30:393-406. 
Scheffel L, et al. Semantic features and lexical semantic knowledge

APPENDIX. Features in Each Condition of High, Mid and Low Importance and Relevance. The Numbers are the Relevance Rate (RR) and the Importance Rate (IR)

\begin{tabular}{|c|c|c|c|c|c|c|c|c|c|}
\hline & HI $3.0-4.0$ & & & $\mathrm{MI} 2.0-2.9$ & & & LI $0-1.9$ & & \\
\hline $\mathrm{HR}$ & & $\mathrm{RR}$ & $\mathbb{R}$ & & $\mathrm{RR}$ & $\mathbb{R}$ & & $\mathrm{RR}$ & $\mathbb{I R}$ \\
\hline $3.0-4.0$ & (Butterfly) colorful & 3.2 & 3.8 & (crayon) paper cover & 3.5 & 2.8 & (apple) red skin & 3.4 & 1.4 \\
\hline & (candle) made of wax & 3.9 & 3.7 & $\begin{array}{l}\text { (diamond) used for engagement } \\
\text { rings }\end{array}$ & 3 & 2.1 & (apple) yellow skin color & 3 & 1 \\
\hline & (carrot) orange & 3.9 & 3.7 & (fox) red & 3 & 2.4 & (candle) smells & 3.1 & 1.4 \\
\hline & (duck) quacks & 3.8 & 3.7 & (moccasin) slippers & 3.3 & 2.2 & (crayon) made by crayola & 3 & 1 \\
\hline & (elephant) has a long trunk & 3.9 & 4.0 & (owl) nocturnal & 3.4 & 2.5 & (pie) fruit & 3 & 1 \\
\hline & (lemon) sour & 3.4 & 3.0 & (rabbit) two long ears & 3.8 & 2.8 & (owl) wise & 3.0 & 0.7 \\
\hline & (pie) crust & 3.9 & 4.0 & (stove) gas & 3.8 & 2.1 & (rabbit) soft fur & 3.2 & 1.7 \\
\hline & (sailboat) sails on water & 3.4 & 3.7 & (stove) heat & 3.2 & 2.5 & (sailboat) jib & 3.1 & 1.7 \\
\hline & (stove) used for cooking & 3.5 & 4.0 & (strawberry) has many seeds & 3.2 & 2.2 & (soap) bar & 3.4 & 1.2 \\
\hline & (trumpet) brass instrument & 3.4 & 3.5 & (trumpet) has three valves & 3.5 & 2.9 & (soap) liquid & 3 & 1.1 \\
\hline MR & (apple) has a core & 2.5 & 3.8 & (candle) melts & 2.5 & 2.8 & (apple) used to make cider & 2.4 & 1 \\
\hline $2.0-2.9$ & (blouse) buttons & 2.4 & 3.8 & (carrot) source of vitamin A & 2.4 & 2.6 & (candle) tallow & 2.6 & 1.6 \\
\hline & (butterfly) four wings & 2.4 & 3.7 & (elephant) trumpets & 2.6 & 2.5 & (rabbit) small & 2.5 & 1 \\
\hline & (carrot) root & 2.5 & 4 & (fox) similar to a dog & 2.7 & 2.6 & (carrot) underground & 2.5 & 1 \\
\hline & (crayon) many colors & 2.6 & 3.4 & (lemon) juice & 2.5 & 2.4 & (diamond) cut glass & 2.5 & 1.4 \\
\hline & (duck) water bird & 2.6 & 4 & (owl) eats mice & 2.5 & 2.6 & (elephant) In circus & 2.5 & 1 \\
\hline & (elephant) large/big animal & 2.3 & 4 & (rabbit) twitchy nose & 2.6 & 2.6 & (soap) shower & 2.5 & 1 \\
\hline & (fox) soft fur & 2.5 & 3.2 & Soap (slippery) & 2.3 & 2.4 & $\begin{array}{l}\text { (moccasin) made by native } \\
\text { Americans/Indians }\end{array}$ & 2.5 & 1.3 \\
\hline & (lemon) tart & 2.4 & 3.5 & (stove) electric & 2.6 & 2.3 & (strawberry) short cake & 2.6 & 1.3 \\
\hline & (trumpet) mouthpiece & 2.6 & 3.6 & (strawberry) low growing plant & 2.6 & 2.6 & $\begin{array}{l}\text { (trumpet) hold it with both } \\
\text { hands }\end{array}$ & 2.3 & 1 \\
\hline LR & (apple) can eat the skin & 1.6 & 3.7 & (blouse) worn with suit & 1.2 & 2.1 & (candle) emergency light & 1.5 & 1.4 \\
\hline $0-1.9$ & (blouse) garment & 1.1 & 3.6 & (diamond) valuable & 1.5 & 2.5 & (carrot) good in soups & 1.3 & 1 \\
\hline & (butterfly) lays eggs & 1.2 & 3.5 & (duck) dark meat & 1.7 & 2.6 & (crayon) pencil like & 1.2 & 1.4 \\
\hline & (candle) burns & 1.4 & 3.5 & (fox) bright eyes & 1.7 & 2.6 & (diamond) bright & 1.6 & 1 \\
\hline & (diamond) dig from mines & 1.7 & 3.5 & (moccasin) footwear & 1.7 & 2.7 & (elephant) wild & 1.3 & 1.3 \\
\hline & (duck) feathers & 1.5 & 4 & (owl) small ears & 1.5 & 2.5 & (fox) beautiful & 1.2 & 1 \\
\hline & (elephant) four feet & 1.1 & 4 & (rabbit) animal & 1.2 & 2.6 & $\begin{array}{l}\text { (lemon) grows in California } \\
\text { or Florida }\end{array}$ & 1.5 & 1.6 \\
\hline & (owl) bird & 1.3 & 3.5 & (sailboat) float & 1.7 & 2.4 & (pie) sweet & 1.3 & 1 \\
\hline & (stove) appliance & 1.1 & 3.6 & (stove) made of metal & 1.3 & 2.6 & (soap) different scents & 1.4 & 1.6 \\
\hline & (trumpet) musical instrument & 1.6 & 4 & (trumpet) treble notes & 1.2 & 2.1 & (strawberry) jelly & 1.7 & 1.2 \\
\hline
\end{tabular}

